\title{
UN MODELLO DI SANTITÀ FEMMINILE ALLA CORTE DEI MEROVINGI: PER UN APPROCCIO STORICO-RELIGIOSO ALLA FIGURA DI GERTRUDE BADESSA DI NIVELLES
}

1. Premessa. È noto che da alcuni anni lo studio sulla santità femminile è oggetto di una significativa intensificazione, soprattutto per via della diffusione di quella nuova tendenza culturale, originariamente di matrice californiana, detta di gender, che, talvolta, si carica di rivendicazioni socio-politiche non pienamente congrue con un'imparziale indagine storico-religiosa. Tuttavia, se ricondotta entro gli strumenti derivanti dalle nuove acquisizioni mediante approcci critici e metodologici che siano sia storici sia filologici, una lettura analitica sull'opera delle donne nel contesto che qui più ci interessa, ossia quello della santità occidentale altomedievale - più esattamente precarolingia - può indubbiamente aiutare a porre ancor più in chiaro, tra le altre cose, l'apporto offerto dalle Sante alla costruzione dell'Occidente cristiano.

Quello dell'agiografia consacrata alle donne negli anni che prepararono la Rinascita Carolingia è un fenomeno religioso tale da meritate studi che ne approfondiscano, sul piano culturale ancor più che storico, l'influenza sulla mentalità e l'immaginario popolari; del resto, l'agiografia è essenzialmente genere metastorico (non negazione della storia, ma trasfigurazione di essa), sicché è a questo che ci si deve oltremodo rivolgere, affinché siano individuate e comprese le dinamiche ideologiche che informarono le singole età dell'uomo europeo lungo quei secoli che - è assodato (pur nella diversità di visione, basterebbero da sé, ed è già parecchio, i lavori di Peter Brown ${ }^{1}$, Franco

${ }^{*}$ Dr Fausto Iannello* - dottore di ricerca $(\mathrm{PhD})$ in Storia delle Religioni, collabora (honorary fellow) alla Cattedra di Storia del Cristianesimo presso il Dipartimento di Civiltà Antiche e Moderne dell’Università degli Studi di Messina; faustoiannello@gmail.com.

${ }^{1}$ Cf. P. Brown, La formazione dell'Europa cristiana. Universalismo e diversità. 200-1000 d.C., trad. it. M. Sampaolo (The Rise of Western Christendom. Triumph and Diversity. A.D. 200-1000, Oxford 2003²), Roma - Bari 2006. Tra i numerosi contributi del grande storico irlandese, su questi concetti rimangono fondamentali e preziosi quelli donati durante le sue letture all'Università di Yale nell'ottobre del 1986, riunite in P. Brown, The End of the Ancient Other World: Death and Afterlife between Late Antiquity and the Early Middle Ages (The Tanner Lectures on Human Values), Yale University 1986. 
Cardini ${ }^{2}$, Bruno Luiselli³ - formarono quell'identità cristiana dell'Europa che Novalis, mille anni dopo, avrebbe vagheggiato nella sua fuggevole vigilia. Ed è stato proprio Peter Brown che più d'altri ha rimarcato quanto preziose e fondamentali permangano, su un piano culturale, laddove "cultura" sia intesa quale Weltanschauung, figure come la principessa badessa Gertrude, in vista di una ridefinizione del concetto di "formazione dell'Occidente", vale a dire di una nuova realtà, culturale e non, si capisce, geografica, giacché negli anni della fondazione di Nivelles, quando una santa "barbara" trapassa al suo Dio proprio nel giorno del dies natalis di quel Patrizio che aveva convertito terre mai prima d'allora romanizzate, germoglia e cresce la consapevolezza di una identità specifica nelle regioni nord-occidentali d'Europa ${ }^{4}$.

In un volume a più mani dedicato al simbolismo della natura nel Cristianesi$\mathrm{mo}^{5}$, avevo rilevato e discusso, comparativamente, proprio le strette connessioni tra l'Irlanda e l'ambiente religioso e culturale generatosi attorno alla figura della nostra aristocratica merovingia, attraverso la specifica comparazione del racconto della navigazione verso la Britannia di alcuni monaci che, invocando la santa, riescono a rendere vano l'attacco del maligno manifestatosi nelle sembianze di un mostro marino. La Vita sanctae Geretrudis - come in quella sede avevo già sostenuto - costituisce una fonte preziosa, unica in taluni casi, per le informazioni sulla famiglia e l'ambiente di Pipino di Landen, nonché per la conferma dei profondi rapporti culturali e religiosi tra la cristianità irlandese e l'occidente precarolingio negli specifici anni del cosiddetto "miracolo irlandese", e, ancora, per la ricezione nel continente di tradizioni e fenomeni religiosi già tipici dell'agiografia insulare altomedievale e dell'universo monastico occidentale.

Alla luce del percorso intrapreso con quel contributo, in questa sede si vuole principalmente ricostruire e valutare l'azione di Gertrude di Nivelles

${ }^{2}$ Cf. F. Cardini, Le radici cristiane dell'Europa. Mito, storia, prospettive, Rimini 1997.

${ }^{3}$ Cf. B. Luiselli, La formazione della cultura europea occidentale, Roma 2003.

${ }^{4}$ Cf. Brown, La formazione dell'Europa cristiana, p. 327-328.

${ }^{5} \mathrm{Cf}$. F. Iannello, Sul retaggio e le fonti irlandesi della "Vita Geretrudis": il santo e la "belua marina", in: Il simbolismo degli elementi della natura nell'immaginario cristiano, a cura di M.A. Barbàra, Atti della Accademia Peloritana dei Pericolanti. Classe di Lettere, Filosofia e Belle Arti 85, Supplemento 1, Napoli 2010, 173-196.

${ }^{6}$ Vedi soprattutto Le miracle irlandaise, éd. H. Daniel-Rops, Paris 1956; B. Bischoff, Il Monachesimo irlandese nei suoi rapporti col Continente, in: Il Monachesimo nell'Alto Medioevo e la formazione della civiltà occidentale, Settimane di Studio del Centro Italiano di studi sull'Alto Medioevo 4, Spoleto 1957, 121-138, L. Bieler, Ireland. Harbinger of the Middle Ages, trad. ingl. (Irland. Wegbereiter des Mittelalters, Olten 1961), London - New York-Oxford 1963. Bruno Luiselli (La formazione della cultura europea occidentale, p. 507, n. 144), forse più d'altri, è riuscito a rendere con le giuste parole cosa debba intendersi con siffatto "miracolo": "il miracolo è nel fatto che l'Irlanda, sempre rimasta fuori del mondo romano e dunque mai romanizzata, assorbì, grazie alla cristianizzazione e al monachesimo, cultura di tradizione romana, e questa stessa cultura esportò in Britannia e nel Continente, contribuendo così, da protagonista, al processo di formazione della cultura europea occidentale". 
per delinearne e stabilirne quegli elementi che possano concorrere a porre in chiaro quanto la nostra donna si attagli a quella paradigmatica identità di santa altomedievale che fa di se stessa il veicolo per quei modelli di santità cristiana universale da custodire e traghettare verso quelli, poi canonici e standardizzati, del Basso Medioevo, allorché, riprendendo delle felici immagini di una illustre specialista di materia agiografica, la santità si colora al femminile, creandosi una sorta di inflazione agiografica ${ }^{7}$. Benché la santità delle donne appaia spesso come una santità adespota, essa "costituì un indubbio 'omaggio' della Chiesa alla donna e ai valori che esprimeva e rappresentava nella società anche come sposa e madre: nei secoli del pieno Medioevo il fenomeno fu in costante crescita fino a raggiungere numericamente quote mai più toccate" . Forse qualcuno potrebbe obiettare che quella femminile sia una santità originale, o persino comparabile, rispetto a quella maschile, giacché a quest'ultima successiva nella tradizione agiografica, ma questo significherebbe non considerare come funzionali per la fede le differenze di sensibilità che sono legate anche alle tipicità derivanti dal sesso.

Ad oggi, tra i non molti studi accademici specifici su Gertrude, eccellono particolarmente quelli di Bonnie Effros, che via via saranno qui citati, l'introduzione e lo splendido commento di Paul Fouracre e Richard A. Gerberding alla loro stessa traduzione inglese della $\mathrm{Vita}^{9}$, e finanche gli interventi sparsi di Peter Brown, malgrado una "stasi accademica" che, da circa tre anni, è interrotta da interventi afferenti perlopiù ai cosiddetti gender studies. È specialmente il caso di un importante e ben documentato saggio di Courtney Luckhardt ${ }^{10}$, che ha il merito di aver rilevato l'interesse nei riguardi dei peregrini da parte di Gertrude, la quale, infatti, benché non itinerante, nella sua stabilitas si fece testimone di un kerygma finalizzato specialmente alla promozione, mediante l'ospitalità, di viaggi dalle diverse finalità (sociali, culturali, missionarie, ascetiche), a usufruire dei quali furono soprattutto le comunità di Irlandesi ivi giunt. Questa funzione, peraltro, si attaglia comparativamente

${ }^{7}$ Cf. A. Benvenuti Papi, La santità al femminile: funzioni e rappresentazioni tra medioevo ed età moderna, in: Les fonctions des saints dans le monde occidental (III ${ }^{e}-X I I{ }^{e}$ siècle). Actes du colloque. (Roma, 27-29 octobre 1988), Roma 1991, École Française de Rome, 467-488. Sulle concezioni di santità al femminile nel Medioevo in genere, vedi, dell'eminente esperto André Vauchez, il capitolo "La santità al femminile", in: idem, Esperienze religiose nel Medioevo, Roma 2013, 97-109. Per un quadro del mondo religioso femminile nella Gallia franca, vedi J.M.H. Smith, The Problem of Female Sanctity in Carolingian Europe c. 780-920, "Past and Present" 146 (1995) 3-37 e C. Urso, Donne e potere nella Galia merovingia e carolingia, Catania 2000.

${ }^{8}$ C. Urso, La donna e la Chiesa nel Medioevo. Storia di un rapporto ambiguo, "Annali della Facoltà di Scienze della Formazione" (Catania) 4 (2005) 67-99, spec. p. 97.

${ }^{9}$ Cf. Vita Sanctae Geretrudis (The Life of St. Geretrud), in: P. Fouracre - R.A. Gerberding, Late Merovingian France: History and Hagiography 640-720, Manchester - New York, 1996, 319-326.

${ }^{10}$ Cf. C. Luckhardt, Gender and Connectivity: Facilitating Religious Travel in the Sixth and Seventh Centuries, "Comitatus” 44 (2013) 29-53. 
a quella di patrona dei viaggiatori, che, soprattutto nell'Europa settentrionale, ha generato la credenza di una Gertrude con funzioni psicopompe nella sua ipostasi di custode e ostessa di una locanda dove transitano le anime durante la prima notte successiva alla morte ${ }^{11}$.

Gertrude, per di più, ospitò e assisté orfani, vedove, poveri, prigionieri e schiavi liberati ${ }^{12}$. Il saggio della Luckhardt palesa una sapiente capacità di interpretazione di meccaniche socio-religiose: utilizzando il genere come un obiettivo, la studiosa analizza la natura delle reti femminili di collegamento religioso e di comunicazione nell'Alto Medioevo (secoli VI-VII), comparando le figure dell'irlandese ancestrale Brigida di Kildare, di Radegonda di Poitiers, moglie del merovingio Clotario I, e della nostra Gertrude, e dunque dimostrando come le donne facilitarono i contatti attraverso il dialogo e l'interazione con gli altri, parenti, se donne, religiosi, pellegrini e precettori, se uomini. Queste relazioni hanno così permesso alle donne di svolgere un ruolo cruciale nella formazione di interconnessioni culturali e religiose, eterogenee, in spazi pure limitati: "Like Mediterranean people in the sixth and seventh centuries, north-western Europeans had a diversity of religious experience that brought them into contact with other Christian regions, a geographical fluidity that allowed for communication and contact over long and short distances. Female saints were a key part of these connections, facilitating the movement of people and ideas"13.

Altrettanto demarcanti, e ancora in un ambito di gender studies, sono i risultati delle ricerche di Susan Wade ${ }^{14}$, corroboranti l'unicità del modello femminile incarnato dalla pulzella di Nivelles. La studiosa rileva e discute la tipologia di tonsura portata da Gertrude, che permane senza riscontri nell'agiografia femminile del tempo ed espressione di una visione e di costumi religiosi caratteristici della aristocrazia franca. Per volontà della madre Itta, Gertrude, dall'età di ventuno anni, recava infatti un taglio utilizzato dagli uomini in ambito regale o monastico, scelto per proteggerla dal matrimonio forzato e quindi consentirle al meglio la fondazione del monastero:

${ }^{11}$ Cf. G. De Santillana (in coll. con H. Von Deschend), Il mulino di Amleto. Saggio sul mito e sulla struttura del tempo, trad. it. A. Passi (Hamlet's Mill, An Essay on Myth and the Frame of Time, Boston 1969), Milano 1983, p. 246, n. 2 e p. 344.

${ }^{12}$ Cf. Vita Geretrudis 3; De virtutibus 8; Additamentum Nivialense de Fuilano 450 (quest'ultimo numero corrisponde alla pagina dell'edizione di Bruno Krusch in MGH, i cui dati saranno forniti tra breve, insieme a quelli delle altre opere or ora citate).

${ }^{13}$ Luckhardt, Gender and Connectivity, p. 53.

${ }^{14} \mathrm{Cf}$. S. Wade, Gertrude's tonsure: an examination of hair as a symbol of gender, family and authority in the seventh-century Vita of Gertrude of Nivelles, "Journal of Medieval History" 39 (2013) fasc. 2, 129-145. Della Wade, vedi anche Miraculous Seeing and Monastic Identity: Miracles of the Visual from the Monasteries of Lobbes and Nivelles, Ph.D. Dissertation, New York University 2007, che esamina prevalentemente le visioni della santa nelle agiografie qui in oggetto e la loro fortuna per tutto il Medioevo. 
"Ut non violatores animarum filiam suam ad inlecebras huius mundi voluptates per vim raperent, ferrum tonsoris arripuit et capillos sanctae puellae ad instar coronae abscisit"15.

2. Fonti e notizie. Per ricostruire storicamente le gesta di Gertrude, abbiamo a disposizione ben tre fonti a lei coeve, che poi sono anche le uniche dirette sulle origini della fondazione di Nivelles ${ }^{16}$. La più importante è notoriamente la Vita sanctae Geretrudis, tramandataci da codici risalenti pure al sec. VIII ${ }^{17}$, che fu compilata intorno al 670 da un monaco irlandese testimone oculare delle azioni della santa fino al letto di morte ${ }^{18} \mathrm{e}$, dunque, da annoverarsi tra i più antichi agiografi $\mathrm{d}^{\prime} \operatorname{Irlanda}^{19}$. Se ne sono conservate due tradizioni: la prima non solo è più antica, ma è anche priva delle correzioni e delle emendazioni di età carolingia. Si capisce, pertanto, che risulta essere lo strumento privilegiato, più affidabile e utile in questa sede e, in genere, per le ricerche sulla santa. Questa Vita, relativamente all'obiettivo qui prefisso, risulta inoltre una fonte molto importante per la ricostruzione sia dei rapporti tra una cristianità "di margine", com'è quella merovingia delle Gallie settentrionali, con tradizioni periferiche, sia con tradizioni e visioni continentali della santità al tempo ben radicate nel mondo ecclesiastico mediterraneo.

Di seguito, si riportano schematicamente la tradizione manoscritta e le edizioni e le traduzioni fin qui pubblicate.

\section{Vita S. Geretrudis}

Manoscritti: Versio A: Montpellier, Bibliothèque Interuniversitaire, Section médecine, H 55, olim Boherianus B 8, sec. VIII,; Paris, Bibliothèque Nationale de France, latin, 11748, f. 141, X Sankt Gallen, Kantonsbibliothek Vadiana, 556, 291-326, X-XI München, Bayerische Staatsbibliothek, Codices latini monacenses, 4618, f. 75-89, Bened. n. 118, sec. XI; Versio B: Paris, Bibliothèque Nationale de France, latin, 5593, f. 30, IX; Bruxelles, Bibliothèque Royale de Belgique, 7882, X-XI.

Edizioni: L. Surius, De probatis sanctorum historiis, II, Köln 1578, 302-306 (compendio di Vita e Virtutes); J.-G van Ryckel, Historia sanctae Gertrudis: Principis virginis primae Nivellensis, Bruxellis 1637; G. Henschenius, Acta

${ }^{15}$ Vita S. Geretrudis 2, ed. B. Krusch, MGH Scriptores Rerum Merovingicarum II, Hannoverae $1888,456$.

${ }^{16}$ Per un quadro storico di questa sede in età medievale, vedi J.J. Hoebanx, L'Abbaye de Nivelles des origins au XIV siècle, Brussels 1952.

${ }^{17}$ Cf. Vita S. Geretrudis 7, ed. Krusch, p. 449.

${ }^{18}$ Cf. ibidem 7, ed. Krusch, p. 448; K. Hughes, The Church in Early Irish Society, London 1966, 684. Sui problemi cronologici vedi Fouracre - Gerberding, Late Merovingian France, p. 301-329.

${ }^{19}$ Cf. J.F. Kenney, The Sources for the Early History of Ireland. Ecclesiastical. An Introduction and Guide, (prima edizione Dublin 1929) repr. with addenda by L. Bieler, New York 1966, 503-504. 
Sanctorum, Martii, II, Antverpiae 1668, 594-599; J. Mabillon, Acta Sanctorum ordinis S. Benedicti, Saeculum II, Parisiis 1669, 463-472; J. Ghesquierus et C. Smetius, Acta Sanctorum Belgii selecta, III, Brixellae 1785, 144-266; B. Krusch, Vita sanctae Geretrudis, MGH Scriptores Rerum Merovingicarum II, Hannover - Hahn 1888, 447-464 (è quest'ultima che qui seguo).

Traduzioni: La vie et les oeuvres de s. Gertrude vierge et abbesse de l'ordre de s. Benoist, trad. J. Mege, Paris, 1687, e quella inglese già citata di Fouracre e Gerberding, curatori (327-329) anche di quella del già menzionato, e sul quale si tornerà, Additamentum Nivialense de Fuilano (ed. B. Krusch, MGH Scriptores rerum Merovingicarum, IV, 449-451, 449), redatto da un monaco di Nivelles nel VII secolo (mss. Bruxelles, Bibliothèque Royale de Belgique, 207, f. 59, XIII, e Paris, Bibliothèque Nationale de France, latin, 2768A, f. 73, XI). Della cosiddetta "versione B" e delle Virtutes possediamo la traduzione inglese di J. McNamara e J. Halborg in: Sainted Women of the Dark Ages, London 1992.

Di una Vita Geretrudis Tertia, sec. XI (BHL 3494) sappiamo che il manoscritto più antico risale al sec. XII (Bruxelles).

Mentre scrivo non si hanno dati di una traduzione in lingua italiana; esiste tuttavia una traduzione della riscrittura curata in francese da Jacqueline Bouette de Blémur nel suo L'année Benedictine, ou Les vies des Saints de l'Ordre de Saint Benoist, pour tous les jours de l'année, 7 tomes, Paris 1667-1673: La vita di s. Gertrude vergine, e badessa di Nivella, in Anno benedettino, ovvero Vite de' santi dell'Ordine di s. Benedetto distribuite per ciaschedun giorno dell'anno. Opera tradotta dal franzese nello idioma italiano. Tomo secondo, che contiene li mesi di marzo, e aprile, Venezia 1727, 144-148.

Dopo la Vita, il trittico delle fonti dirette include le Virtutes (De virtutibus, quae facta sunt post discessum beate Gertrudis abbatisse) e la loro Continuatio (Virtutum sanctae Geretrudis continuatio), sempre edite insieme. Le prime sono fondate su tradizioni orali e furono redatte per iniziativa di Agnese, terza badessa di Nivelles (ca. 670-), alla fine del VII secolo ${ }^{20}$.

Le Virtutes, che nei codici A della Vita la seguono sempre, sono continuazioni della vita, scritte nel 783, così tràdite:

\section{Virtutes Geretrudis}

Manoscritti: Montpellier, Bibliothèque Interuniversitaire, $\mathrm{H}$ 55, VIII, olim Boherianus B 8; München, Bayerische Staatsbibliothek, Codices latini monacenses, 4618 (Bened. n. 118), f. 75-89, XI; München, Bayerische

${ }^{20}$ Cf. J.-J. Hoebanx, L'abbaye de Nivelles, des origines au XIV siècle, Bruxelles 1952, 31; C. Holvoet, Sainte Gertrude de Nivelles: la "Vita Tripartita" du XI siècle et les techniques de réécriture, Mémoire de Licence - Histoire, Université Libre de Bruxelles, Bruxelles 2006, 18. 
Staatsbibliothek, Codices latini monacenses, 18854 (Teg. N. 854), f. 179 (continuatio Virtutum), XI; Oxford, Bodleian Library, Bodley, 8687, Feller. I, XII; Paris, Bibliothèque Nationale de France, latin, 11748, f. 141, X; Sankt Gallen, Kantonsbibliothek Vadiana, 556, 291-326, IX-X; Zwettl, Stiftsbibliothek, Codex S. Crucis Mellicensi.

Edizioni: De virtutibus, quae facta sunt post discessum beate Geretrudis abbatisse (anno 690 ca.), ed. B. Krusch, MGH Scriptores rerum Merovingicarum II, Hannover 1888, 464-474.

Traduzioni: J. McNamara - J. Halborg, Sainted Women of the Dark Ages, London - Durham, 1992, 229-234.

Gertrude fu dunque l'aristocratica rampolla di una casata merovingia appena assurta agli onori regali ${ }^{21}$ : la si ricorda quale zia di Pipino di Héristal, quindi prozia di Carlo Martello, in quanto figlia di san Pipino di Landen (575-640), il quale, senza soluzione di continuità, fu maestro di palazzo rispettivamente di Clotario II (584-629), Dagoberto I (610-639, regno 622-639) e Sigeberto III (ca. 630-656), e regnante sul trono di Austrasia dal 633 al 656 ${ }^{22}$; unico figlio maschio di Pipino di Landen fu Grimoaldo I, anch'egli maggiordomo, dal 643 al 657, e re d'Austrasia (633-656); la madre di Gertrude, santa Itta (o Iduberga $\uparrow$ 652), intorno al 647 fu fondatrice di quel monastero doppio di Nivelles (oggi nel Brabante vallone, tra Liége e Tournai; fiammingo Nijvel; latino Nivialcha, Niviala, Niviella) del quale la figlia sarà badessa.

Gertrude, nata nel 626 ca., muore, trentatreenne, molto probabilmente nel 659, in quel 17 marzo $^{23}$ che aveva già segnato il trapasso di san Patrizio, così adempiendosi una profezia dell'irlandese Ultano ${ }^{24}$. L'idea del monastero doppio fu dovuta probabilmente sia al modello già sperimentato da Patrizio e perfezionato da Brigida ${ }^{25}$ sia a quello più vicino introdotto proprio tra $\mathrm{i}$ Merovingi

${ }^{21}$ La combinazione di nobiltà di sangue e virtù ascetiche e apostoliche caratterizzò diverse sante nell'Occidente germanico altomedievale; tra le altre, basti ricordare le regine Radegonda di Poitiers (sec. VI), Batilde di Neustria (VII), Eteldreda di Ely (VII), Sexburga di Ely (VII-VIII), Matilde di Ringelheim (X) e le badesse Hathumoda di Gandersheim (sec. IX), Edith di Wilton (sec. X) e Adelaide di Willich (sec. XI). In merito si veda Smith, The Problem of Female Sanctity in Carolingian Europe, passim.

${ }^{22}$ Fondamentale, per una storia religiosa del periodo in oggetto, rimane Y. Hen, Culture and Religion in Merovingian Gaul, AD 481-751, Leiden - New York 1995.

${ }^{23}$ Vita Sanctae Geretrudis 7, ed. B. Krusch, MGH Scriptores rerum Merovingicarum II, 447464, spec. p. 462; pure P. Grosjean, Chronologie de S. Feuillen, AnBol 75 (1957) 379-393, spec. p. 390. Pochi anni dopo, nel 700 ca., e ancora a Nivelles, saranno redatte le Virtutes Geretrudis (De virtutibus, quae facta sunt post discessum beate Geretrudis abbatisse, ed. B. Krusch, MGH Scriptores rerum Merovingicarum II 464-474).

${ }^{24}$ Cf. Vita S. Geretrudis 7; vedi anche L. Gougaud, Les Saints irlandais hors d'Irlande, Louvain - Oxford 1936, 187.

${ }^{25}$ Cf. J. Ryan, Irish monasticism, origins and early development, Dublin - Cork, 1931, 80. 
da Burgundofara di Eburiac (meglio nota come Fara), la figlia del maestro di palazzo Cagnerico che, intorno al 620, su indicazione dell'irlandese Colombano, era stata la fondatrice della celebre abbazia di Faremoutiers, nel nord della Francia $^{26}$. In questi luoghi, una comunità maschile si affiancava a una femminile per le questioni liturgiche, spirituali, didattiche e, in genere, per sostenere tutte quelle occupazioni che le donne non potevano espletare.

La Vita ci tramanda che Gertrude, ancora quattordicenne, già decisa a consacrarsi alla vita religiosa, rabbiosamente ${ }^{27}$ negò la sua mano al figlio di un dux Austrasiorum durante un banchetto dato in onore del re Dagoberto dal padre ${ }^{28}$, che morì appena dopo, intorno $\mathrm{al} 640^{29}$. Le pressioni furono sì violente e inaspettate da causare un rifiuto altrettanto radicale e le conseguenti offese e ingiustizie degli altri notabili ${ }^{30}$, che spinsero Itta, col concorso del figlio Grimoaldo - costui anche per comprensibili mire politiche ${ }^{31}-$ a scegliere la vita monastica ${ }^{32}$. Da qui, la madre, su consiglio di sant'Amando, fece edificare il monastero di Nivelles, del quale fu la prima badessa. La figlia amata si dedicò in tonsura e velo alla formazione e essa medesima promosse gli studi ecclesiastici ottenendo e diffondendo codici provenienti da Roma e affidando l'istruzione a esegeti irlandesi:

"Erga eclesiastica studia vasa summo studio pastoralem habebat curam, et per suos nuntios, boni testimonii viros sanctorum patrocinia vel sancta volumina de urbe Roma et de transmarinis regionibus gignaros nomine ad docendum divini legis carmina, sibi et suis meditandum, Deo inspirante, meruisset habere" ${ }^{33}$.

La madre morì nel 652, sessantenne, nello stesso monastero e fu sepolta nella basilica di san Pietro apostolo ${ }^{34}$. Fece costruire altri edifici nei pressi del mo-

${ }^{26}$ Cf. Ionas Bobiensis, Vita S. Columbani II 7, ed. B. Krusch, MGH Scriptores Rerum Germanicarum XXXVII, Hannover - Leipzig 1905, 240-243.

${ }^{27}$ Cf. Vita S. Geretrudis 1, ed. Krusch, p. 454-455: "At illa quasi furore repleta, respuit illum cum iuramento et dixit, nec illum nec alium terrenum nisi Christum dominum volebat habere sponsum". Sul furore della Santa, vedi C. Peyroux, Gertrude's furor: Reading Anger in an Early Medieval Saint's Life, in: Anger's Past: the social uses of emotion in the Middle Ages, ed. B. Rosenwein, Cornell 1998, 36-58.

${ }^{28} \mathrm{Si}$ veda su questo episodio la comparazione con altre sante, specialmente con la principessa franca Rictrude di Marchiennes (613 ca.-688), di K. Ugé, The Legend of Saint Rictrude: Formation and Transformations (Tenth-Twelfth Century), in: Anglo-Norman Studies. XXIII. Proceedings of the Battle Conference 2000, ed. J. Gillingham, Woodbridge - Rochester 2001, 281-297.

${ }^{29}$ Cf. Vita S. Geretrudis 2, ed. Krusch, p. 455.

${ }^{30}$ Cf. ibidem, ed. Krusch, p. 455-457.

${ }^{31}$ Cf. Additamentum, ed. Krusch, p. 451.

${ }^{32}$ Cf. Vita S. Geretrudis 2, ed. Krusch, 456; vedi anche R. Le Jan, Convents, Violence, and Competition for Power in Seventh-Century Francia, in: Topographies of Power in the Early Middle Ages, ed. F. Theuws - M.B. De Jong - C. Van Rhijn, Leiden 2001, 243-269.

${ }^{33}$ Vita S. Geretrudis 2, ed. Krusch, p. 457.

${ }^{34}$ Cf. ibidem, ed. Krusch, p. 447. 
nastero e ricevé aiuti ${ }^{35}$. Gertrude demandò allora ogni occupazione alle consorelle, per dedicarsi alle "res divinae e agli obscura allegoriae misteria" 36 .

3. Gertrude e gli Irlandesi. Nivelles, che negli anni appena successivi alla morte di Gertrude avrà tra i suoi abati l'irlandese Suibhne ${ }^{37}$, fu subito inclusa nella paruchia Fursei ${ }^{38}$, straordinaria realtà umana e culturale che spiega la massiccia e decisiva presenza di ecclesiastici ibernici in siffatti territori. Si trattava di una sorta di confederazione di chiese e celle fondate e rette da Fursa, dai suoi consanguinei, nonché dai suoi più stretti confratelli, nelle Gallie nord-orientali ${ }^{39}$ a partire dal $648^{40}$, quando Fursa, lasciata l'Irlanda nel $630 \mathrm{ca}$., sarebbe arrivato nel continente dal monastero di Cnoberesburgh (Suffolk, EstAnglia $)^{41}$. A succedergli nell'opera, tanto nell'Est-Anglia quanto, dopo il 650, nelle Gallie, saranno i suoi fratelli Foillán (Foillianus/Foelanus: uterinus Fursei frater $^{42}$ ), anche'egli abate di Cnoberesburgh, ucciso verso il $655^{43}$, e il già menzionato Ultán (Ultanus), abbate di Péronne fino al 686, data della sua dipartita ${ }^{44}$. Dopo aver fondato le abbazie di Lagny-sur-Marne (Latiniacum) ${ }^{45}$ e di Péronne

${ }^{35}$ Cf. ibidem 3, ed. Krusch, p. 458.

${ }^{36}$ Ibidem. Tra le monache era anche Gudula, nobile originaria del Brabante, battezzata da Gertrude e oggi santa patrona di Bruxelles.

${ }^{37}$ Cf. Hughes, The Church in Early Irish Society, p. 94.

${ }^{38}$ Ibidem, p. 82.

${ }^{39}$ In Piccardia sorge ancora Frohen-le-Grand (ora nel comune di Frohen-sur-Authie), l'antica Forshem, "casa di Fursa", con una cappella del santo un tempo meta di pellegrini, cf. M. Stokes, Three Months in the Forests of France: a pilgrimage in search of vestiges of the Irish saints in France, London 1895, 175.

${ }^{40}$ Cf. T.W. Charles-Edwards, Early Christian Ireland, Cambridge 2000, 318.

${ }^{41}$ Cf. Grosjean, Chronologie, p. 392; Hughes, The Church, p. 93; H. Mytum, The Origins of early Christian Ireland, London - New York 1992, 77, S. Hamann, St Fursa, the genealogy of an Irish saint - the historical person and his cult, "Proceedings of the Royal Irish Academy" 112C (2011) 147-187.

${ }^{42}$ Additamentum Nivialense de Fuilano, ed. Krusch, p. 449. Vedi anche Martyrology of Oengus ([sec. IX], ed. W. Stokes: Félire Óengusso Céli Dé. The Martyrology of Oengus the Culdee, London 1905, 230) al 31 ottobre: "Faelán, .i. martir fratrer fursu".

${ }^{43}$ Vedi Vita et Miracula Fursei dal Codex Salmanticensis c. 7, ed. W.W. Heist, Vitae Sanctorum Hiberniae ex Codice olim Salmanticensi nunc Bruxellensi, Bruxelles 1965, 52; anche Beda, Historia Anglorum III 19, ed. B. Colgrave - R.A.B. Mynors, Oxford 1969 (repr. 1991), 274, dove si danno i nomi di altri due monaci al seguito di Fursa: Gobán e Dícuill, cf. Betha Fursa, ed. W. Stokes: The Life of Fursa, "Revue Celtique" 25 (1904) 385-404, spec. p. 398.

${ }^{44}$ Cf. Gougaud, Les saints, p. 98 ss.; Grosjean, Chronologie, p. 379 ss. Per le notizie concernenti i viaggi e le fondazioni dei tre fratelli, oltre alla Vita Fursei (Vita Fursei abbatis Latiniacensis, ed. B. Krusch, MGH Scriptores Rerum Merovingicarum IV 435-440), si veda il già citato Additamentum de Fuilano. Per altri documenti sui tre santi, Kenney, The Sources, p. 501-505.

${ }^{45}$ Vita Fursei 9, ed. Krusch, 438: "monasterium in loco nuncupante Latiniaco construxit"; Virtutes Fursei 13, ed. B. Krusch, MGH Scriptores Rerum Merovingicarum IV, 440-449, spec. p. 444: 
(Parona civitas Galliae ${ }^{46}$ ), chiamata Perrona Scottorum perché retta da soli abati irlandesi per tutto il sec. VII e oltre ${ }^{47}$, Fursa vi morirà intorno al 649/650 stando agli Annali dell'Ulster ${ }^{49}$, l'ultimo abate irlandese di Péronne, Maenán, abbas civitatis Fursei, morì nel $779^{50}$. La Vita Fursei ${ }^{51}$, pure inserita in codici contenenti scritti patriciani (ms. Arras, Bibl. Munic. 450, sec. XII), è tràdita da numerosi codici che altresì ne conservano una tarda redazione gaelica (XVII sec. $)^{52}$. Secondo altre fonti, Fursa, di stirpe regale, era originario della stessa terra di un altro celebre santo irlandese, Brendano di Clonfert ( $\dagger$ ca. 577), nato appunto nel Munster, e forse pronipote dello stesso ${ }^{53}$. Dal celebre santo navigatore egli ricevé il battesimo, per poi essere istruito proprio a Clonfert ${ }^{54}$, da dove avrebbe recato sul continente le reliquie di Patrizio e del maestro ${ }^{55}$. In più, Fursa visitò Inis maccu-Chuinn (Inchiquin), sul Lough Corrib ${ }^{56}$, ricordata come sede di un monastero fondato da Brendano verso il $550^{57}$ per accogliere pellegrini ${ }^{58}$. I suoi fratelli, ospitati entrambi a Nivelles per volonta di Gertrude, riceveranno da Itta delle terre nella vicina Fosses ${ }^{59}$ (nel IX sec. detta ancora Monasterium Scottorum ${ }^{60}$ ), dove la struttura monastica presto fondata sarà retta da Ultán ${ }^{61}$.

In realtà, colui che per primo promosse la fondazione di Nivelles fu il padre spirituale di Itta, il vescovo itinerante Amando di Maastricht $(\dagger 674 / 679)^{62}$,

\footnotetext{
"sanctus Furseus Latiniacum suis minibus construxit, beatusque Erchenaldus Perronam cum festinatione praeparavit" (Erchinoaldo era maestro di palazzo di Neustria per Clodoveo II).

${ }^{46}$ Vita Cuannathei 9 e 10, ed. Heist, p. 49.

${ }^{47}$ Péronne rimase un' istituzione prevalentemente ibernica fino alla sua distruzione per mano dei Norvegesi nell'880, cf. K. Hughes, The Golden Age of Early Christian Ireland, in: The Course of Irish History, ed. T.W. Moody - F.X. Martin, Cork 1967, 76-90, spec. p. 84-85.

${ }^{48}$ Cf. Grosjean, Chronologie, p. 392-393; Hamann, St Fursa, the genealogy of an Irish saint, p. 148, passim.

${ }^{49}$ Cf. Annals of Ulster 779.2, ed. S. Mac Airt - G. Mac Niocaill, Dublin 1983, 235.

${ }^{50}$ Per un recente quadro storico sulle origini della missione irlandese nelle regioni in questione: C. Mériaux, Gallia irradiata. Saints et sanctuaires dans le nord de la Gaule du haut Moyen Âge, Stuttgart 2006, 38-42.

${ }^{51}$ Cf. Vita Fursei, ed. Krusch. Vedi anche la versione salmanticense, ed. Heist, p. 37-50.

${ }^{52}$ Cf. Betha Fursa, ed. Stokes, "Revue Celtique" 25 (1904) 385-404.

${ }^{53}$ Cf. P. Ó Riain, Sanctity and politics in Connacht c. 1100: the case of St Fursa, "Cambridge Medieval Celtic Studies" 17 (1989) 1-14.

${ }^{54}$ Cf. J. Dunn, The Brendan problem, "The Catholic Historical Review" 6 (1921) 395-477, spec. p. 409.

${ }^{55}$ Vedi l'edizione della cosiddetta Vita II Fursei (XI sec.), ASanc Ianuarii I 44-54.

${ }^{56} \mathrm{Cf}$. Martyrology of Oengus 45.

${ }^{57}$ Cf. M. Archdall, Monasticon Hibernicum, or an history of the abbies, priories, and other religious houses in Ireland..., I-II, Dublin 1786, repr. 1876, qui II 215.

${ }^{58} \mathrm{Cf}$. Dunn, The Brendan problem, p. 409.

${ }^{59} \mathrm{Cf}$. Additamentum 451.

${ }^{60}$ M. Richter, Ireland and her neighbours in the seventh century, Dublin 1999, 131.

${ }^{61}$ Cf. Gougaud, Les Saints, p. 98; Grosjean, Chronologie, p. 393.

${ }^{62}$ Cf. Vita S. Geretrudis 2.
} 
sostenuto da Dagoberto I nella missione nelle Fiandre ${ }^{63}$ e per tre anni affiancato da Giona di Bobbio nella diocesi di Tournai, dove quest'ultimo redasse gran parte della Vita Columbani ${ }^{64}$. Secondo le fonti locali, l'episcopato di Amando a Maastricht (oggi diocesi di Liège) cadde negli anni 648-651, per cui si può stabilire che la fondazione di Nivelles avvenne tra il 646 e il 647, allorché Gertrude doveva avere circa $20 \mathrm{anni}^{65}$. Dalla cittadina belga di Saint Amand provengono i più antichi manoscritti continentali della Vita Brigitae di Cogitosus (ca. 650), laddove il culto di Colombano († 615) e Fursa è attestato nel continente già nella seconda metà del sec. VII ${ }^{66}$. Della sola Brigit sono attestati circa centoventi siti cultuali nelle terre tra la Senna e la Mosa, comprese Fosses e Nivelles, che ne ospitano entrambe una celebre cappella ${ }^{67}$. Ulteriori conferme provengono anche da costumi e liturgie caratterizzanti questa località per tutto il Medioevo: a Nivelles le erano informate la benedizione dell'acqua il $17 \mathrm{di}$ marzo, secondo una tradizione già seguita in Irlanda ${ }^{68}$, e invocazioni in antichi rituali per infermi ${ }^{69}$. In merito alla tradizione patriciana, la testimonianza più antica sul continente proviene proprio da Péronne, ossia il carme esametrico noto come Epigramma di Cellano, l'abate di Péronne († 706) successore di Ul$\operatorname{tano}^{70}$. La più antica attestazione continentale della Vita Columbae (688-696) di Adomnán (628-704) è nel manoscritto dell'abbazia di Echternach risalente agli inizi del sec. VIII ${ }^{71}$, ora alla Bibliothèque nationale de France ${ }^{72}$. Nel corso del sec. VIII la Vita arrivò nel continente anche nella copia di Dorbéne di Iona $(† 713)$, che ha lasciato chiare sue tracce in codici di Fosses, Nivelles e della vicina Saint Amand $^{73}$; grazie all'interazione tra i monaci irlandesi di Péronne

${ }^{63}$ Cf. H. Platelle, Le temporal de l'abbaye de Saint-Amand, des origines à 1340, Paris 1962, 38; J.-M. Picard, Church and politics in the seventh century: the Irish exile of King Dagobert II, in: Ireland and northern France AD 600-850, ed. idem, Dublin 1991, 27-52, 34-35.

${ }^{64} \mathrm{Cf}$. un commento di A. De Vogüe, in: Jonas de Bobbio, Vie de saint Colomban et de ses disciples, ed. idem, Abbaye de Bellefontaine (Bégrolles-en-Manges) 1988, 21; J.-M. Picard, Adomnán's "Vita Columbae" and the cult of Colum Cille in continental Europe, "Proceedings of the Royal Irish Academy" 98C (1998) 1-23, spec. p. 5-6.

${ }^{65} \mathrm{Si}$ veda A. Dierkens, Saint Amand et la foundation de l'abbaye de Nivelles, „Revue du Nord” 68 (1986) 326-341.

${ }^{66}$ Cf. Picard, Adomnán's, p. 2.

${ }^{67} \mathrm{Cf}$. Gougaud, Les saints, p. 26-30.

${ }^{68}$ Cf. ibidem, p. 28.

${ }^{69} \mathrm{Cf}$. P. Grosjean, Le souvenir de S. Killan à Nivelles, AnBol 75 (1957) 373-377.

${ }^{70}$ Cf. ed. L. Traube, Vorlesungen und Abhandlungen, 3, München 1920, 107. Vedi Gougaud, Les saints, p. 148; L. Bieler, The Life and the Legend of St. Patrick, Dublin 1949, 121; E. Coccia, La cultura irlandese precarolingia: miracolo o mito?, „Studi Medievali” 8 (1967) 257-420, spec. p. 318-320.

${ }^{71}$ Cf. D. Ó Cróinín, Rath, Melsigi, Willibrord and the earliest Echternach manuscipts, "Peritia" 3 (1984) 17-49.

${ }^{72}$ Cf. J.-C. Müller, Trois manuscrits liturgiques de l'abbaye d'Echternach à Paris, in: Abteistadt Echternach, ed. P. Schritz - A. Hoffmann, Luxembourg 1981, 202-206.

${ }^{73}$ Cf. Picard, Adomnán's, p. 4-5. 
e gli ambienti franco-colombaniani, una copia della versione di Dorbéne giunse dall'Irlanda nella stessa Péronne, se non proprio a Fosses ${ }^{74}$. Nel suddetto manoscritto di Echternach è peraltro contenuto il Calendario di Willibrord, testimone capitale per l'associazione della triade "Patrizio - Brigit - Colum Cille" in area continentale almeno dalla fine del sec. VII ${ }^{75}$. Sia Péronne sia Iona erano tra loro in contatto grazie alla mediazione del monastero irlandese di Louth (Leinster), incluso nella paruchia di san Fursa ${ }^{76}$, giunto nell'Est-Anglia direttamente da Louth ${ }^{77}$. Non poche connessioni tra tradizione e documenti patriciani e Louth sono state rintracciate e discusse ${ }^{78}$; qui è opportuno rilevare che il successore di Mochta a Louth, Gorman $(\uparrow 753 / 757)^{79}$, era padre di Torbach († 808) - comarba (successore) di Patrizio giacché vescovo di Armagh - che negli anni 807-808 fece redigere il ben noto Liber Ardmachanus ${ }^{80}$.

4. Il transitus. Avendo appreso "per revelationem divinam" $" 11$ la prossimità della fine del suo percorso terreno, Gertrude, "omni honori renuntians, consultis monachis monialibusque" 82 , scelse, tra queste ultime, colei che le sarebbe successa, la nipote ventenne Vulfetrude (Wulfetrud), figlia di Grimoaldo ${ }^{83}$. Dopo tre mesi trascorsi in preghiera e astinenza, inviò un monaco al vicino monastero di Fosses per apprendere da Ultano in quale giorno sarebbe morta. Costui rispose che sarebbe avvenuto in quello stesso giorno, ovvero all'ora sesta di quella stessa domenica, 17 del mese di marzo, aggiungendo che bisognava prepararsi con le solennità ed esser lieti perché proprio Patrizio, che,

${ }^{74}$ Cf. Picard, Church and politics, p. 31-35.

${ }^{75}$ Cf. Kalendarium S. Willibrordi, ed. H.-A. Wilson: The Calendar of St. Willibrord, London 1918; vedi J.-M. Picard, The marvellous in Irish and continental saints 'Lives of the Merovingian period, in: Columbanus and Merovingian Monasticism, ed. H.B. Clarke - M. Brennan, Oxford 1981, 91-103, spec. p. 99.

${ }^{76}$ Cf. Picard, Adomnán's, p. 5.

${ }^{77}$ Cf. Charles-Edwards, Early Christian, p. 318. Così nel Mart. of Oengus (4 settembre), ed. Stokes, p. 201: "Ultan was elected into the abbacy of Mochta in Louth, and before him Fursa had been put thereout".

${ }^{78} \mathrm{Cf}$. C. Doherty, The cult of St Patrick and the politics of Armagh in the seventh century, in: Ireland and the Northern France, AD 600-850, ed. J.-M. Picard, Dublin 1991, 53-94, spec. p. 53 e 56-57.

${ }^{79}$ Cf. Annals of Tigernach, ed. W. Stokes, "Revue Celtique" 16 (1895) 374-419; 17 (1896) 6-33, 119-263, 337-420; 18 (1897) 9-59, 150-197, 267-303, spec. p. 258; Annals of the Four Masters = Annala Rioghachta Eireann: Annals of the Kingdom of Ireland by the Four Masters, from the Earliest Period to the Year 1616. Edited from MSS in the Library of the Royal Irish Academy and of Trinity College Dublin with a Translation and Copious Notes, ed. J. O'Donovan, 7 voll., Dublin 1848-1851, qui vol. I, 357 (s.a. 753.6).

${ }^{80} \mathrm{Cf}$. R. Sharpe, Palaeographical considerations in the study of the Patrician documents in the Book of Armagh, "Scriptorium" 36 (1982) 3-28.

${ }^{81}$ Vita S. Geretrudis 6, ed. Krusch, p. 447.

${ }^{82}$ Ibidem.

${ }^{83}$ Cf. ibidem. 
si badi, nella Vita Geretrudis riceve la sua antica menzione in un documento continentale, era già pronto ad accogliere la giovane con i suoi angeli:

"laeta pergat, quia beatus Patricius cum electis angelis Dei et cum ingenti gloria parati sunt eam recipere" $"$.

In questa occasione, Gertrude manifestò una paura che non deve lasciare sorpresi: la massima gloria ultraterrena si accompagna, in Gertrude, alla massima minaccia del male, giacché, sì, avrà paura, ma gioirà rendendo grazie a Dio:

"non timeat neque trepidet de obito suo, sed laeta pergat" $"$.

L'accento posto sulla morte di Gertrude costituisce un topos monastico maschile già attestato dall'antichità, quello del giorno della morte inteso come giorno capitale, ossia dies natalis, esso stesso regola di vita monastica nella forma di racconto esemplare: in realtà, l'attesa formalmente parossistica della morte è, per Gertrude, un exemplum e, insieme, un atto iniziatico, giacché, come è stato brillantemente asserito, "ciò che si gioca nel momento del transitus del santo fondatore non è soltanto l'atteso passaggio dal quaggiù all'aldilà, ma anche il pericoloso passaggio da un carisma personale a un'istituzione fatta per durare, da un ideale sempre e sempre più idealizzato dall'agiografia a una pratica quotidiana, a un necessario inserimento nella Chiesa e nella società" ${ }^{86}$. E così che nel Cristianesimo la realtà storica e quella escatologia si ricompongono, annullando il contrasto, tutto moderno e sconosciuto all'agiografia tradizionale, di storia orizzontale e storia verticale, per cui la storia dell'uomo si fa da essa stessa storia della sua salvezza ${ }^{87}$.

Il sarcofago marmoreo nel quale fu posta la santa fu da subito utilizzato come fonte battesimale colmato d'olio per sanare i malati ${ }^{88}$ e simultaneamente cominciarono a intensificarsi i miracoli intorno al sarcofago. La riapertura si compì l' 8 del mese di luglio dell'anno 1293 per direttiva della badessa Elisabeth di Bierbais e fu presto seguita da un inquietante avvenimento, ovverosia la morte della stessa appena pochi mesi dopo, quasi a corroborare delle superstizioni aleggianti in quei luoghi. Sorprendente lo stato di conservazione della santa:

\footnotetext{
${ }^{84}$ Ibidem 7, ed. Krusch, p. 463.

${ }^{85}$ Ibidem. Vedi in merito le osservazioni di Peter Brown nelle già citate Tanner Lectures di Yale, p. $49-50$.

${ }^{86}$ J. Delarun, La morte dei santi fondatori. Da Martino a Francesco, in: Un gallo ad Asclepio. Morte, morti e società tra antichità e prima età moderna, a cura di A.L. Trombetti Pugliesi, Bologna 2013, 407-425, 407-408.

${ }^{87}$ Cf. O. Cullman, Cristo e il tempo. La concezione del tempo e della storia nel Cristianesimo primitivo, trad. it. B. Ulianich (Christus und die Zeit. Die urchristliche Zeitund Geschichtsauffassung, Zurich 1946), Bologna 1965, 74-93.

${ }^{88}$ Cf. B. Effros, Merovingian Mortuary Archaeology and the Making of the Early Middle Ages, Berkeley - Los Angeles - London 2003, 221-222; della stessa autrice, cf. anche Caring for Body and Soul. Burial and Afterlife in the Merovingian World, University Park Pennsylvania 2002, 24-25, 35-36.
} 
con l'esumazione, infatti, si scoprì un corpo ancora intatto, con l'eccezione di soli tre denti, probabilmente trafugati come reliquia dopo la prima traslazione nel $1059^{89}$. La paura diffusasi portò a una nuova riapertura soltanto nel $1848^{90}$.

Dopo la morte di Wulfetrud, all'età di trent'anni ${ }^{91}$, la tradizione monastica della famiglia sarà custodita da Begga, sorella di Gertude ${ }^{92}$.

5. Funzionalismi. Alla luce degli eventi e dei temi esposti e discussi, ritengo che il testo agiografico conosciuto come Vita Geretrudis sia strutturato nelle seguenti sequenze e/o nuclei narrativi.

1. Prologo: familiae Geretrudis e congregatio Nivialensis.

2. Capitolo 1: radicale rifiuto della forzata richiesta di matrimonio $\rightarrow$ manifestazione pubblica della vocazione $\rightarrow$ unione con Cristo.

3. Cap. 2: morte di Pipino; missione e apostolato di Amando a Nivelles; definizione del ruolo pastorale e dell'identità spirituale della vedova Itta (sobrietà, castità); costruzione del monastero; "persecuzione" e opposizione $^{93}$ da parte dei vicini; iniziazione e voti di Gertrude (tonsura, velo); esercizio delle virtù cristiane ${ }^{94}$

${ }^{89}$ Cf. J. Lestocquoy, The Reliquary of St. Gertrude at Nivelles, “The Burlington Magazine" 77 (1940) 162-164.

${ }^{90} \mathrm{Su}$ queste notizie e, in generale, i fatti e gli elementi mortuari concernenti la figura di Gertrude, vedi: J. De Borchgrave d'Altena, Des statuettes de la châsse de sainte Gertrude, "Annuaire de la Société Archéologique et Folclorique de Nivelles" 13 (1942) 283-291; R. Lemaire, Les avant-corps de Sainte-Gertrude à Nivelles, Antwerpen 1942; R. Hanon De Louvet, L'inspection des ossements de Sainte Gertrude eut-elle lieu en 1292 ou en 1293?, "Annales de la Société Archéologique et Folklorique de Nivelles et du Brabant Wallon" 17 (1952) 249-254; C. Donnay-Rocmans, La châsse de Sainte Gertrude à Nivelles, "Gazette des Beaux-Arts" ser. VI 58 (1961) 185-202; J. Mertens, Recherches archéologiques dans l'abbaye mérovingienne de Nivelles, "Archaeologia Belgica" 61 (1962) 108-111; B.K. Young, Exemple aristocratique et mode funéraire dans la Gaule mérovingienne, "Annales: Economie, Sociétés, Civilisations" 41 (1986) 379-407; B. Effros, Symbolic Expressions of Sanctity: Gertrude of Nivelles in the Context of Merovingian Mortuary Custom, "Viator" 27 (1996) 1-10; idem, Merovingian Mortuary Archeology, p. 21ss. Su questi fatti locali di Nivelles si vedano le seguenti fonti: B. Des Hayes, Cathalogue des abbesses de Nivelle, et des choses et gestes les plus remarquables escheus en leur administration abbatiale, depuis Ste Gertrude jusqu'au commencement du 17e siècle (1652), Bibliothèque Royale (Brussels) MS 16596, ff.33r ss.; E. Bauget, Notice sur un manuscrit comprenant l'histoire des abbesses de Nivelles, "Compte-rendu des Séances de la Commission Royale d'Histoire" 6 (1843) 179-184; J.F. Foppens, Bibliotheca belgica sive virorum in Belgio vitam, scriptisque illustrium, I, Brussels 1739, 117.

${ }^{91}$ Cf. Vita S. Geretrudis 6, ed. Krusch, p. 460.

${ }^{92}$ Cf. Virtutes 10. Una Vita Beggae risale alla fine sec. XI (BHL 1083); vedi l'eccellente ricerca di S. Leclère, L'abbaye d'Andenne, VII ${ }^{e}-X I I I^{e}$ siècle. De la fondation aristocratique par sainte Begge à la transformation en chapitre noble, Mémoire de Licence, Université Libre de Bruxelles 2011.

${ }^{93}$ Cf. Vita S. Geretrudis 2, ed. Krusch, p. 456: "Quales iniurias vel ignobilitates et penuriae propter nomen Christi supradicta famula Christi cum filia sua perpessa est, scribere longum est".

${ }^{94} \mathrm{Cf}$. ibidem: "[...] morum continentiam, mentes sobrietatem, verborum moderationem seni- 
4. Cap. 3: transitus di Itta; passaggio alla fase mistica: orazioni, digiuni, veglie notturne; rivelazione degli obscura allegoriae misteria per mezzo dello Spirito santo; ampliamento delle strutture monastiche e cura dell'ospitalità ${ }^{95}$.

5. Capp. 4-5: primi miracoli (mirabilia); potere di intercessione, vittoria sul maligno.

6. Cap. 6: logoramento corporale, visioni $\rightarrow$ annuncio della morte; ritiro ed investitura del successore (Vulfetrude).

7. Cap. 7: transitus (riti mortuari: vestizione) - profezia di Ultano, reductio a un santo maggiore (Patrizio), letizia dinanzi alla morte, veglia, due testimoni per le cure spirituali (uno è l'autore) del monastero, effusione di profumi dal corpo, benefici per le orazioni svolte sulla sepoltura della santa.

La giovane Gertrude esprime funzionalmente numerosi e incisivi tratti di una santità cristiana universale, certo, ma nondimeno originale, che ne fanno, pertanto, un modello autentico. Nelle sue vicende biografiche si distinguono: la "persecuzione" già implicita nel tentativo di matrimonio forzato, la decisa e prematura "uscita dal mondo", la presenza di un autorevole anziano maestro fin dalla giovinezza, ruolo svolto da Amando e quindi dalla madre, con profonde analogie all'aite irlandese (ingl. fosterfather, un tutore che fungeva anche da padre spirituale, riservato esclusivamente ai figli della nobiltà locale $)^{96}$, i miracoli concessi in vita, il dono di ricevere visioni e annunci dello Spirito Santo, dunque la prescienza e la conoscenza dei misteri cristiani. Con una sobrietà che colpisce, l'autore, poi, offre di Gertrude un ritratto delicato e discreto (valga più di tutti, e si confronti, quello relativo alle sue virtù, già sopra riportato). Si aggiungano il dominio sulle tentazioni e gli assalti del maligno, anche attraverso sue ipostasi ferine (Vita 6) ${ }^{97}$, l'ascetismo unito all'apostolato, alla pastorale e alla carità (elemosina, accoglienza, liberazione degli schiavi, fondazioni religiose e culturali, famiglia monastica).

Credo che da quanto detto si possa trarre un preciso percorso sequenziale di tipo iniziatico nella vita spirituale di Gertrude: contemptus mundi, vocazione,

\footnotetext{
lem antecessit aetatem. Erat autem caritate predita, vultu pulcra, sed pulcrior mente, castitate integra, aelimosinis larga, ieuniis et orationibus provida, infirmis ac senibus pia, iuvenibus vero disciplina extitit strenua".

${ }^{95}$ Cf. ibidem 3, ed. Krusch, p. 458: "Item sanctorum eclesias et alia praecipua aedificia ex fundamentis construxit et orfanis, viduis, peregrinis alimonia cotidiana cum omni largitate ministravit".

${ }^{96}$ Vedi F. Kelly, A Guide to Early Irish Law, Dublin 1988, 86-90; T.M. Charled-Edwards, Early Christian Ireland, Cambridge 2004 (prima ed. 2000), 115-116. Sulle relazioni tra i religiosi e gli aristocratici presso i Merovingi, vedi A.-M. Helvétius, L'organisation des monastères féminins à l'époque mérovingienne, in: Female vita religiosa between Late Antiquity and the High Middle Ages. Structures, development and spatial contexts, ed. G. Melville - A. Müller, Wien - Berlin 2011, 151-169.

${ }^{97}$ Vedi il mio già citato articolo sulla Vita Geretrudis.
} 
formazione, ascesi, miracoli, missione, indizione di una tradizione, nascondimento, morte gloriosa.

A una "dimensione secolare", la santa lascia riconoscersi in un modello costruito con il concorso dei seguenti caratteri: magisterialità, signoria, mecenatismo, istruzione e pedagogia, solidarietà, sofferenza fisica, zelo, letizia, il tutto sostenuto - ed è forse questo il punto più decisivo - dal superamento del modello androcentrico e ginecocentrico fondato su un rapporto di complementarietà e/o di intercambiabilità tra i religiosi di entrambi i sessi, vale a dire una collaborazione molto stretta tra donne e uomini che di fatto costituiva il superamento delle fratture della specializzazione dei ruoli tra uomini e don$\mathrm{ne}^{98}$. La santificazione di Gertrude è ottenuta nella dimensione quotidiana della famiglia, in una condizione di stabilitas, attraverso passaggi di rottura e di destabilizzazione della condizione femminile ordinaria, nella famiglia di origine, prima, in quella monastica, poi.

Gertrude, generalmente venerata in ambito rurale quale protettrice contro le invasioni dei topi, quindi, per converso, signora dei gatti ${ }^{99}$, esercita funzioni ben testimoniateci iconograficamente: il libro (la fede, l'istruzione), un modello di chiesa o di ospedale (le fondazioni religiose e i centri di accoglienza), il boccale (il fonte battesimale miracoloso nella sua cripta a Nivelles), il pellegrino e, appunto, i topi ${ }^{100}$. La sua più antica raffigurazione è dedicata invece proprio all'episodio storico del taglio dei capelli da parte della madre (Passionale di Stoccarda, anno 1130 ca., f. 57, Landesbibliothek, Stuttgart $\left.{ }^{101}\right)$.

Quasi in una vichiana eterogenesi, i modelli di santità, riducibili ad una unica fonte oltremondana e a una universale prospettiva escatologica, riescono plurimi e sussistenti da se medesimi nella loro orizzontale specificità e nel loro contributo alla storia delle nazioni, dunque delle culture. Del resto, la santità è una vocazione universale: "haec est enim voluntas Dei, sanctificatio vestra" (1Thess 4, 3; cfr. Eph 1, 4). "Le donne" scrive Sofia Boesch Gajano "sono il soggetto storico che trae maggiori benefici dalle fonti agiografiche [...]. Una

${ }^{98}$ Come rileva A. Vauchez nella sua Prefazione a Benedetto XVI, Donne nel Medioevo. Il genio femminile nella storia del popolo di Dio, Genova 2011, 10.

${ }^{99}$ Vedi il saggio di J. Berchtold La tradition médiévale de la «femina muscipula» et l'iconographie de «sainte Gertrude aux rats» dans les Hortuli anima edu XVI siècle, in: idem, Des rats et des ratiere. Anamorphoses d'un champ métaphorique de saint Augustine à Jean Racine, Genève 1992, specialmente le pagine 73 ss. Vedi anche E. Zurli, Un mantello tempestato di topi: il singolare carisma di Gertrude di Nivelles, in: Aa.Vv., Donne sante, sante donne, Viterbo 2007, 81-93 (nello stesso volume si offre anche una ricostruzione storica del fenomeno della santità femminile presso l'aristocrazia merovingia: Valente Bacci, Le regine sante merovingie, p. 13-32).

${ }^{100}$ Cf. L. Goosen, Dizionario dei santi. Storia, letteratura, arte e musica, trad. it. M.C. Coldagelli - S. Contarini - R. Novità - F. Paris (Van Afra tot de Zevenslapers, Nijmegen 1992), Milano 2002, 213-214.

${ }^{101}$ Cf. Das Stuttgarter Passionale, ed. A. Boeckler, Augsburg 1923. Vedi anche J. Tibbetts Schulenburg, Forgetful of their sex. Female sanctity and society, ca. 500-1000, Chicago 1998, 132, tav. 12. 
storia che, nella conoscenza del passato, voglia recuperare integralmente la presenza delle donne nel loro rapporto dialettico con il maschile trova nelle testimonianze agiografiche una miniera inesauribile di dati sulla loro posizione nella società e nella famiglia, sul loro modo di concepire e vivere l'esperienza religiosa"102. Sembrerebbe non accidentale che "la prima «vera immagine» di Cristo che compare nella cultura occidentale, cioè il telo della Veronica, è collegata dalla leggenda ad una figura femminile", episodio "in cui il Messia rompe il tabù religioso dell'impurità mestruale - lasciandosi toccare dalla donna - che era alla base dell'evitazione delle donne nella cultura ebraica"103.

Gertrude fu un modello di santità al femminile per quelle badesse e monache in genere che ne seguirono l'esempio - alcune delle quali ricordate in questo lavoro - e nello stesso tempo agì come prototipo e archetipo di quel fenomeno chiamato Adelsheilige, termine con il quale si vuol rendere e intendere quel processo di germanizzazione della Cristianità occidentale individuabile nelle Vitae dei secoli VII e VIII attraverso l'attribuzione dei valori e delle virtù dell'aristocrazia franca alle monache poi canonizzate ${ }^{104}$. André Vauchez ha ben sintetizzato i punti fondanti dell'Adelsheilige, ritenendolo scaturito da un incontro tra il desiderio di autocelebrare il proprio lignaggio da parte di dinastie giunte all'apice del potere e la gratitudine che monaci e monache desideravano ardentemente manifestare alle loro benefattrici ${ }^{105}$.

Affido la conclusione di questo scritto a Régine Pérnoud, prendendo in prestito alcune sue parole, nette quanto pacate, che, a mio avviso, riescono a esprimere il senso più autentico della santità femminile nel Medioevo, individuandone specificità e grandezza senza la necessità di appelli alle esacerbanti inquietudini socio-culturali di una qualsivoglia imperante ideologia: "Costituiscono una creazione armoniosa ed equilibrata, questi monasteri duplici la cui originalità ci colpisce e ci fa intravedere una civiltà atta a rovesciare tanti dati che ci sono abituali. Oggi certuni, o meglio certune, pretendono un sacerdozio femminile di cui si sentono come private [...], il ministero sacerdotale appare come un potere, mentre al contrario è un servizio [...]. Pare che allora fosse vivo l'influsso evangelico, e che si considerasse come la donna, assente dalla Cena, fosse la prima che, presso il sepolcro di Cristo, constatasse

${ }^{102}$ S. Boesch Gajano, La santità, Roma - Bari 1999, 52-53.

${ }^{103}$ L. Scaraffia - G. Zarri, Donne e fede. Santità e vita religiosa in Italia, Roma - Bari 2009 (prima ed. 1994), p. XII.

${ }^{104} \mathrm{Si}$ veda, per un quadro storico-religioso del fenomeno, K. Bosl, Der "Adelsheilige”. Idealtypus und Wirklichkeit, Gesellschaft und Kultur im merowingerzeitlichen Bayern des 7. und 8. Jahrhunderts. Geschichte im Spiegel von Geschichtsdeutung, in: Speculum Historiale, ed. C. Bauer - L. Boehm - M. Müller, Freiburg - München 1965, 167-187; J.C. Russell, The Germanization of Early Medieval Christianity. A Sociohistorical Approach to Religious Transformation, New York 1994, passim. Cf. anche J. Kreiner, The Social Life of Hagiography in the Merovingian Kingdom, Cambridge 2014, 15-17.

${ }^{105}$ Cf. Il santo, in: L'uomo medievale, a cura di J. Le Goff, Roma - Bari 1988³ 353-390, spec. p. 364. 
la sua risurrezione, e come ricevesse nella persona di Maria - insieme a tutti gli apostoli - lo Spirito Santo, alla Pentecoste. E inoltre [...] i rapporti che si stabiliscono tra le religiose e i monaci corrispondono a quelli intercorsi tra la Vergine e san Giovanni Evangelista, che "la prese con sé, e per tutta la sua vita fu il servitore premuroso, il figlio amato" 106 .

\section{MODEL KOBIECEJ ŚWIĘTOŚCI NA DWORZE MEROWINGÓW: PRZYCZYNEK HISTORYCZNO-RELIGIJNY NA TEMAT POSTACI KSIENI GERTRUDY Z NIVELLES}

\section{(Streszczenie)}

Autor niniejszego artykułu pragnie omówić historyczno-religijny wpływ wywierany przez św. Gertrudę z Nivelles w świetle nowych trendów kulturowych poruszonych przez tzw. ,gender studies” oraz w kontekście tych lat, które przyczyniły się bardziej niż inne do powstania tak zwanego ,zachodniego chrześcijaństwa”. Przez pryzmat działań młodej ksieni merowińskiej, w kluczowym czasie przygotowującym renesans karoliński, możemy obserwować całkowicie oryginalny model kobiecej świętości.

Key words: Gertrude of Nivelles, Merovingian Church, Early Medieval Monasticism, Irish Missionaries in Continental Europe, Female Sanctity, Gender Studies, Western Christendom.

Parole chiave: Gertrude di Nivelles, Chiesa merovingia, monachesimo altomedievale, missionari irlandesi nell'Europa continentale, santità femminile, studi di genere, cristianità occidentale.

Słowa kluczowe: Gertruda z Nivelles, Kościół merowiński, wczesnośredniowieczny monastycyzm, misjonarze irlandzcy w Europie kontynentalnej, świętość kobieca, gender, chrześcijaństwo zachodnie.

${ }^{106}$ R. Pernoud, I santi nel Medioevo, trad. it. A. Marietti (Les saint au Moyen Âge, Paris 1984), Milano 1986, 103. 